\title{
The use of transdermal optical coherence tomography to image the superficial temporal arteries
}

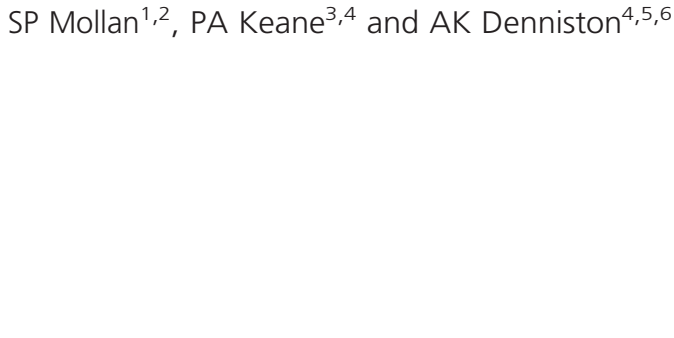

required. Temporal artery biopsy (TAB) is specific, but surgically invasive. Using ultrasound to identify temporal artery oedema ('the halo sign'), stenosis and occlusion has gained popularity. A meta-anaylsis reported the sensitivity and specificity for unilateral halo sign as $68 \%$ and $91 \%$, respectively. ${ }^{2}$ Ultrasound is cheaper, noninvasive, permits longitudinal scanning for disease activity, and can be quicker to organise than TAB but requires experience. ${ }^{2,3}$ Magnetic resonance imaging and angiography ${ }^{4}$ has also been used to image the superficial temporal artery, but access and high expense has limited its widespread use.

Optical Coherence Tomography (OCT) is a noninvasive interferometric optical imaging modality. ${ }^{5}$ Dermal OCT devices are capable of imaging tissues in vivo, including distinct skin layers up to $2 \mathrm{~mm}$ in depth, and the acquisition time is short. ${ }^{6}$ Dermal OCT uses longer infrared wavelengths $(1300 \mathrm{~nm})$ compared with that conventionally used in ophthalmic OCT $(800 \mathrm{~nm})$. Both dermatological and ophthalmic OCT machines have demonstrated the ability to image arterial microvascular structures. ${ }^{7,8}$ To the authors' knowledge, OCT has not yet been used in GCA or health to image the superficial temporal artery (STA).

\section{Materials and methods}

We used a commercially available dermatological multi-beam, Fourier-domain, swept source OCT device (InVivoSight; Michelson Diagnostics, Kent, UK) with a wavelength of 1305 nm (HSL- 2000; Santec Corp, Komaki, Japan), an axial optical resolution of $10 \mu \mathrm{m}$, and lateral optical resolution of $7.5 \mu \mathrm{m}$. The aim was to test the hypothesis that the STA could be imaged using dermal OCT. We scanned eight individuals, all over the age of 50 years: five had a clinical diagnosis of GCA (TAB positive $n=3$; TAB negative $n=2$ ), and
${ }^{1}$ Birmingham NeuroOphthalmology Unit Department of Ophthalmology, University Hospitals Birmingham NHS Trust, Queen Elizabeth Hospital Birmingham, Birmingham, UK

${ }^{2}$ Neurotrauma and Neurodegeneration, School of Clinical and Experimental Medicine, College of Medical and Dental Sciences, The Medical School, The University of Birmingham, Birmingham, UK

${ }^{3}$ NIHR Biomedical Research Centre for Ophthalmology, Moorfields Eye Hospital NHS Foundation Trust, London, UK

${ }^{4}$ University College London, Institute of Ophthalmology, London, UK

${ }^{5}$ Centre for Translational Inflammation Research, School of Immunity and Infection, College of Medical and Dental Sciences, University of Birmingham, Birmingham, UK

${ }^{6}$ Department of Ophthalmology, University Hospitals Birmingham NHS Trust, Queen Elizabeth Hospital Birmingham, Birmingham, UK

Correspondence:

SP Mollan, Birmingham NeuroOphthalmology Unit, Department of Ophthalmology, University Hospitals Birmingham NHS Trust, Queen Elizabeth Hospital Birmingham, Mindelsohn Way, Edgbaston, Birmingham B15 2WB, UK

Tel: +44 (0)789 301 7588; Fax: +44 (0)1216432023 E-mail: soozmollan@doctors.org.uk

Received: 16 November 2015 Accepted in revised form: 14 July 2016

Published online:

14 October 2016 trend ${ }^{1}$ hence a reliable, noninvasive test is 
three were healthy controls with no prior history of GCA symptoms or ophthalmic history (other than cataracts). Three cases are presented here to illustrate the preliminary imaging findings. Ethical approval was obtained and informed consent was taken.

\section{Case reports}

In this proof of concept case series eight individuals were scanned. In all eight (1) the STA could be localized by palpation to align the OCT tracer beam, (2) the STA could be identified on OCT 'free-run' mode, and (3) the STA could then be captured, in part, on 'multi-scan' mode to obtain the desired $6.0 \times 6.0 \mathrm{~mm}$ grid of high-resolution stack of images with $0.1 \mathrm{~mm}$ separation between slices and $2.0 \mathrm{~mm}$ slice depth.

A specific limitation of the technology was found with depth of penetration at around $2 \mathrm{~mm}$, therefore in some cases only the more superficial wall of the artery and lumen were captured. A hyper-reflective band that probably corresponds to elastin within the external elastic lamina, and possibly including the internal elastic lamina, distinguished artery (Figure 1ai-aiii, bi and c) from vein (Figure 1bii). Signal drop out is seen on the images as linear artifact from skin hairs. Where the whole circumference of the artery was captured in the image, a ratio of band thickness (BT) to arterial lumen diameter (ALD) could be calculated (BT : ALD ratio) using the device software.

The following 3 cases were chosen to demonstrate the capability of the device: Case 1 and 3 illustrate the ability to calculate the BT: ALD ratio and Case 2 shows the presence and absence of the hyperreflective band.

\section{Case 1}

A 69-year-old caucasian female presented with bilateral artertic anterior ischemic optic neuropathies. TAB was positive. Figure 1(a) images are from the contralateral side

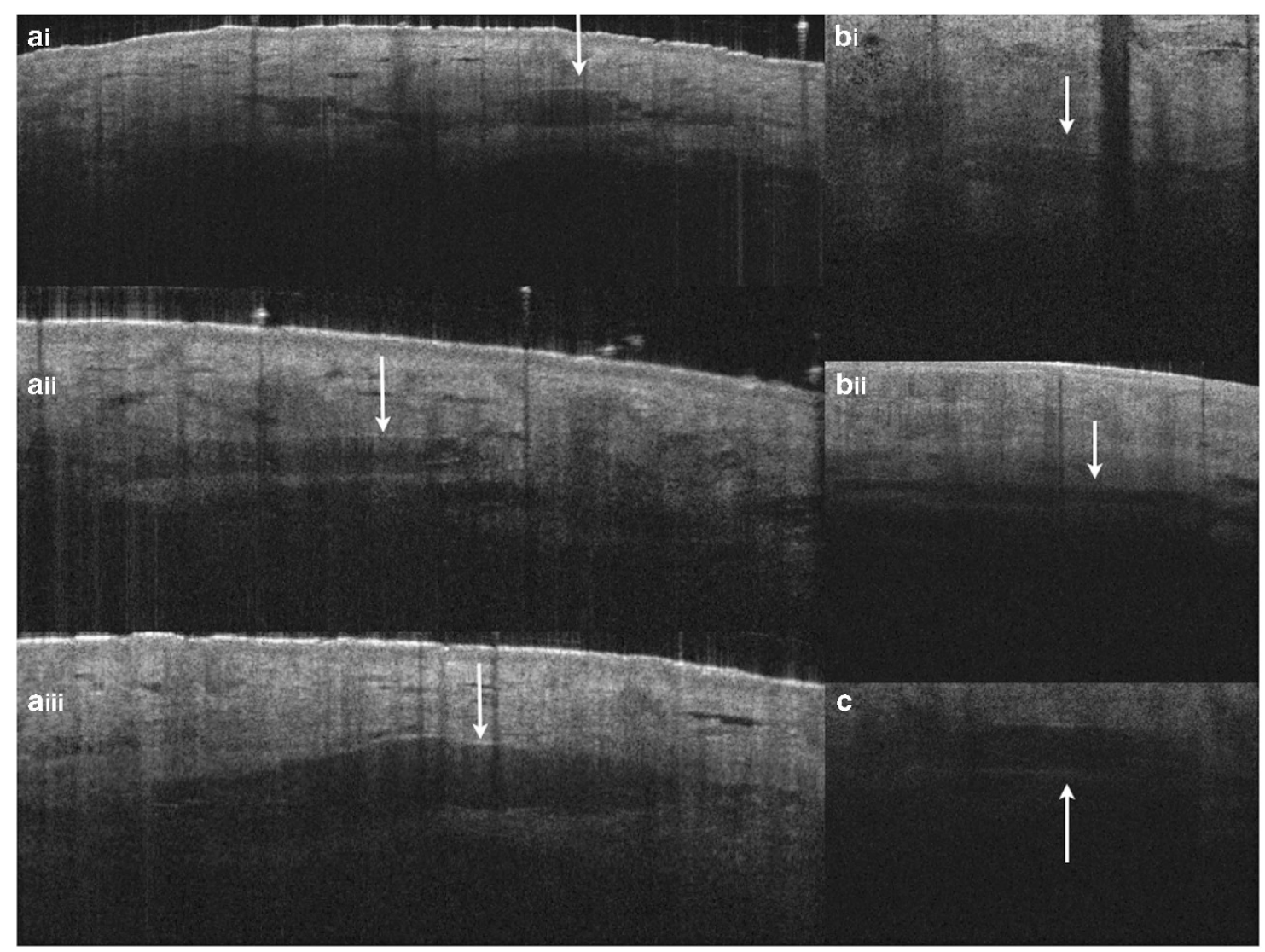

Figure 1 Patient A, a 69-year-old female presented with bilateral artertic anterior ischemic optic neuropathies (TAB positive). Images ai-aii are taken from the side contralateral to the TAB site over 9 months after treatment. The arrow demonstrates a clear hyperreflective band isolating the vessel lumen, this corresponds to the thicker connective tissue wall of an artery rather than vein. Band measures $22 \mu \mathrm{m}$, lumen $240 \mu \mathrm{m}$ (ratio 0.092). Patient B, a 68-year-old male, diagnosed with unilateral artertic anterior ischemic optic neuropathy (TAB positive). Images are taken 4 weeks after initial diagnosis. (bi) Demonstrates the artery proximal to the TAB site; (Bii) Observes a vessel without the hyper-reflective band likely to be a vein. Patient C, a 72-year-old male who has cataracts (control). The arrow demonstrates the clear hyper-reflective band of the artery. Band measures $21 \mu \mathrm{m}$, lumen $213 \mu \mathrm{m}$ (ratio 0.099). 
to TAB site 9 months after diagnosis. The white arrows demonstrate a hyperreflective band isolating the vessel lumen corresponding to the thicker connective tissue wall of an artery rather than vein. $\mathrm{BT}=22 \mu \mathrm{m}, \mathrm{ALD}=240 \mu \mathrm{m}$; BT : ALD ratio 0.092 .

\section{Case 2}

A 68-year-old caucasian male diagnosed with unilateral artertic anterior ischemic optic neuropathy. TAB was positive. Images in Figure 1(b) are taken 4 weeks postdiagnosis, and ongoing corticosteroid treatment. Figure 1 (bi) demonstrates the artery distal to TAB site; Figure 1 (bii) observes a vessel without the hyperreflective band likely to be a vein.

\section{Case 3}

A 72-year-old systemically well caucasian male was reviewed for cataracts (control). The arrow demonstrates the hyper-reflective band of the artery in Figure 1(c). $\mathrm{BT}=21 \mu \mathrm{m}, \mathrm{ALD}=213 \mu \mathrm{m}$; $\mathrm{BT}:$ ALD ratio 0.099.

\section{Discussion}

We have demonstrated that transdermal OCT can identify the STA. The preliminary images presented here need further validation. We found specific limitations of the technology such as the depth of penetration; in some cases only the proximal vessel wall and lumen were captured. Also, artifact from overlying hairs masked the image data that may, in future investigations, be problematic. We did not attempt to scan the parietal branch of the STA and given its position beyond the hairline may not be possible due to hair follicle artifact. Current developments in the technology include enhanced depth imaging, which may see the image data increased by $50 \%$ greater depth. No coupling agent was used and it is probable in future investigations that this may reduce artifact.

Potentially transdermal OCT has a number of advantages over TAB as it is quick and noninvasive, and could be performed serially over time. OCT allows image acquisition by a novice, permits detailed retrospective analysis of all images with extremely high resolution enabling assessment and measurement of structural changes down to a few microns. The axial resolution of OCT images achieved here is less than $20 \mu \mathrm{m}$, up to 10 times higher than conventional ultrasound and magnetic resonance imaging.

As a significant number of patients with large vessel GCA do not have demonstrable temporal artery abnormalities, this could limit the development of dermal OCT for GCA as here we have only demonstrated imaging of the STA. Additionally, current expense of the platform may hinder widespread clinical access for cranial GCA.

These cases provide a proof of concept that transdermal OCT can image parts of the STA. A large-scale validation study of this imaging modality is required to provide evidence that this technique is meaningful in the diagnosis of GCA.

Summary

What was known before

- The diagnosis of GCA is an area of major challenge to rheumatologists, internal physicians, and ophthalmologists.

- Imaging techniques for the STA that are currently being used both for diagnosis and monitoring in GCA include ultrasound and magnetic resonance imaging/magnetic resonance angiography.

- Histopathology from a temporal artery biopsy is still considered the definitive investigation for diagnosis of GCA.

What this study adds

- This is the first report that the superficial temporal artery can be identified and imaged, in part, noninvasively using a commercially available dermal OCT instrument.

\section{Conflict of interest}

The authors declare no conflict of interest.

\section{Acknowledgements}

The VivoSight Scanner was kindly loaned to the department by VivoSight; Michelson Diagnostics, Kent, UK. We would also like to thank both Adam Meekings and Nikki Steadman for their expertise and helpful discussions. PAK has received a proportion of his funding from the Department of Health's NIHR Biomedical Research Centre for Ophthalmology at Moorfields Eye Hospital and UCL Institute of Ophthalmology. The views expressed in the publication are those of the author and not necessarily those of the Department of Health.

\section{References}

1 Mollan SP, Begaj I, Mackie S, O'Sullivan EP, Denniston AK. Increase in admissions related to giant cell arteritis and polymyalgia rheumatica in the UK, 2002-13, without a decrease in associated sight loss: potential implications for service provision. Rheumatology 2015; 54: 375-377. 
2 Arida A, Kyprianou M, Kanakis M, Sfikakis PP. The diagnostic value of ultrasonography-derived edema of the temporal artery wall in giant cell arteritis: a second meta-analysis. BMC Musculoskelet Disord 2010; 11: 44.

3 Czihal M, Zanker S, Rademacher A, Tatò F, Kuhlencordt PJ, Schulze-Koops $\mathrm{H}$ et al. Sonographic and clinical pattern of extracranial and cranial giant cell arteritis. Scand J Rheumatol 2012; 41: 231-236.

4 Rhéaume M, Rebello R, Pagnoux C, Carette S, Clements-Baker $\mathrm{M}$, Cohen-Hallaleh $\mathrm{V}$ et al. Accuracy of high resolution mri of scalp arteries for the diagnosis of giant cell arteritis: results of a prospective study. Arthritis Rheumatol 2014; 66: S390-S391.
5 Huang D, Swanson EA, Lin CP, Schuman JS, Stinson WG, Chang W et al. Optical coherence tomography. Science 1991; 254: 1178-1181.

6 Sattler E, Kästle R, Welzel J. Optical coherence tomography in dermatology. J Biomed Opt 2013; 18: 061224.

7 Yonetsu T, Bouma BE, Kato K, Fujimoto JG, Jang IK. Optical coherence tomography- 15 years in cardiology. Circ J 2013; 77: 1933-1940.

8 Ouyang Y, Shao Q, Scharf D, Heussen FM. An easy method to differentiate retinal arteries from veins by spectral domain optical coherence tomography: retrospective, observational case series. BMC Ophthalmology 2014; 14: 66. 